\title{
Reversible Logic Circuits made of DNA
}

\author{
Anthony J. Genot, Jonathan Bath, Andrew J. Turberfield \\ Department of Physics, Clarendon Laboratory, University of Oxford, Parks Road, Oxford OX1 3PU, United Kingdom
}

Supporting Information Placeholder

ABSTRACT We report reversible logic circuits made of DNA. The circuits are based on an AND gate that is designed to be thermodynamically and kinetically reversible and to respond nonlinearly to the concentrations of its input molecules. The circuits continuously recompute their outputs, allowing them to respond to changing inputs. They are robust to imperfections in their inputs.

Molecular logic circuits have the potential to probe systems of biomolecules and to signal the results of elementary computations on the inputs that they detect. Nucleic acids are both biocompatible and programmable and provide versatile tools with which to monitor biological systems ${ }^{1-6}$. For example, a DNA logic circuit can measure the level of mRNA disease markers and initiate therapeutic action, by producing a drug, if and only if all markers are present $^{7}$. However, the DNA logic circuits demonstrated so far ${ }^{7-18}$ are effectively irreversible, relying on kinetic control of the rates of competing non-equilibrium reactions, typically DNA hydrolysis, controlled by sequence-specific restriction enzymes ${ }^{7,11}$, or DNA hybridization, controlled through the use of single-stranded

'toeholds' to initiate strand-displacement reactions ${ }^{19,20}$. Irreversible reactions can cause problems. Firstly, the computation is performed once and, in general, cannot be redone when inputs change. Secondly, errors are usually also irreversible and can accumulate. Lastly, the state of a circuit depends not only on the present inputs but also on their history. Such hysteresis can lead to spurious results, e.g. in the case of an AND gate whose inputs are sequentially rather than simultaneously present. Irreversible physical mechanisms, including strand displacement ${ }^{21}$ and enzyme restriction $^{22}$, have been proposed as mechanisms for the creation of responsive DNA circuits. Those circuits use energy from covalent or non-covalent chemical reactions to operate away from equilibrium. However, as Bennett pointed out, ${ }^{23}$ reversible chemical reactions can be used to compute with minimal expenditure of energy. In logic circuits that use reversible reactions, the computation and the attainment of equilibrium are equivalent. In such circuits, the activation energies of valid transitions must be low enough to avoid kinetic traps to ensure that the full configuration space is continually sampled. A reversible DNA hybridization mechanism, toehold exchange ${ }^{10,17}$, has been proposed as the basis of implementations of reversible stack machines ${ }^{24}$ and logic circuits $^{25}$. DNA tile self-assembly can be used to compute under near-reversible conditions ${ }^{26}$. Cardelli and Laneve have formalized a reversible concurrent calculus for reversible structures ${ }^{27}$.

Here, we demonstrate experimentally the operation of reversible Brownian ${ }^{23}$ logic circuits based on a simple AND gate, assembled from a single strand of DNA, which is non-linear as well thermodynamically and kinetically reversible. Its inputs are the concentrations of DNA signaling strands. The circuits continuously recompute their outputs as a function of the current inputs: they are therefore responsive.

The reversible AND gate consists of a DNA hairpin (Hp) which equilibrates between ON and OFF conformations in the presence of its inputs, I1 and I2 (Figure 1). The gate contains a domain d2 which is partially double stranded in the OFF state and completely single stranded in the $\mathrm{ON}$ state. In the $\mathrm{ON}$ state, $\mathrm{d} 2$ hybridizes to a molecular beacon reporter ${ }^{28}$ which leads to an increase in the fluorescence emitted by the reporter. When Hp is in the OFF state, $\mathrm{d} 2$ is not accessible and the reporter fluorescence remains quenched. The reporter is designed to unbind quickly, in a time of the order of $100 \mathrm{~s}^{29}$.

Hairpin $\mathrm{Hp}$ is closed by two stems: an external stem opened by hybridization of I1 and an internal stem opened by I2. In order to implement an AND gate, Hp must be ON only when both inputs are present. Hybridization with I1 does not affect the reporter binding domain. Hybridization with I2 frees domain $\mathrm{d} 2$ and allows the reporter to bind (Supporting Information). To prevent input I2 from triggering the output in the absence of I1 (Supporting Information), we engineer cooperativity ${ }^{16}$ in the binding of the inputs: $\mathrm{I} 2$ can bind significantly to $\mathrm{Hp}$ only in the presence of I1. In the discussion below, calculated reaction yields correspond to our experimental concentrations: [Inputs $]=1 \mu \mathrm{M},[\mathrm{Hp}]=[\mathrm{Re}-$ porter] $=0.5 \mu \mathrm{M}$.

Binding of the first input I1 to $\mathrm{Hp}$ is facilitated and stabilized by a single-stranded toehold ${ }^{30,31}$. I1 displaces domain $\mathrm{d} 1$ and opens the external stem (Figure 1). We refer to the resulting structure as Hp.I1. The standard free energy for the binding of I1 to $\mathrm{Hp}$ is approximately $-9.0 \mathrm{kcal} / \mathrm{mol}$ at $25{ }^{\circ} \mathrm{C}$. Under our experimental conditions this is enough to compensate the entropic cost of immobilizing I1, which is of the order of $-\mathrm{RT}^{*} \ln (1 \mu \mathrm{M})=8.15$ $\mathrm{kcal} / \mathrm{mol}$ : Nupack ${ }^{32}$ predicts that about $68 \%$ of $\mathrm{Hp}$ is bound to I1.

The binding of input $\mathrm{I} 2$ is designed to be very weak in the absence of I1. The most stable complex of Hp and I2 (Hp.I.2) is a structure in which $\mathrm{I} 2$ opens the internal stem without breaking the external stem (Figure 1). In this complex the central loop of Hp, consisting of two 5-nt and 3-nt single-stranded domains connected by two double-helical stems, is replaced by a single 15-nt single-stranded domain stretched over the helix formed by hybridization of I2. We estimate the standard free energy for the binding of I 2 to Hp in the absence of I1 to be approximately $-3.7 \mathrm{kcal} / \mathrm{mol}$ (Supporting Information), which includes a contribution of about +4.3 $\mathrm{kcal} / \mathrm{mol}$ corresponding to the difference between the entropic penalties for forming these loops ${ }^{33}$. This is insufficient to compensate for the entropic cost of immobilizing a significant fraction of I2: we estimate that in the presence of $\mathrm{I} 2$, but not I1, less than $1 \%$ of $\mathrm{Hp}$ is converted to Hp.I2.

Binding of I1 and $\mathrm{I} 2$ to $\mathrm{Hp}$ is cooperative: binding of $\mathrm{I} 1$ reduces the entropic penalty for binding I 2 by opening the central loop. : The change in free energy on simultaneous binding of both inputs is more negative than the sum of the corresponding changes on binding of each input separately. The difference between the standard free energy of formation of Hp.I1.I2 from its components $\left(\Delta \mathrm{G}^{0} \approx-17.5 \mathrm{kcal} / \mathrm{mol}\right)$ and the sum of the corresponding free energies of Hp.I1 and Hp.I2 is approximately $-4.8 \mathrm{kcal} / \mathrm{mol}$, corresponding to the difference between the entropic penalties associated with the central loop in Hp and with the d2 loop in Hp.I2 (Supporting Information). When both inputs are present, Nupack predicts that $55 \%$ of $\mathrm{Hp}$ is bound to both $\mathrm{I} 1$ and $\mathrm{I} 2$ and $30 \%$ of $\mathrm{Hp}$ is bound to I1 only. The secondary structure of $\mathrm{Hp}$ is crucial for the cooperativity of the gate. If the central loop 


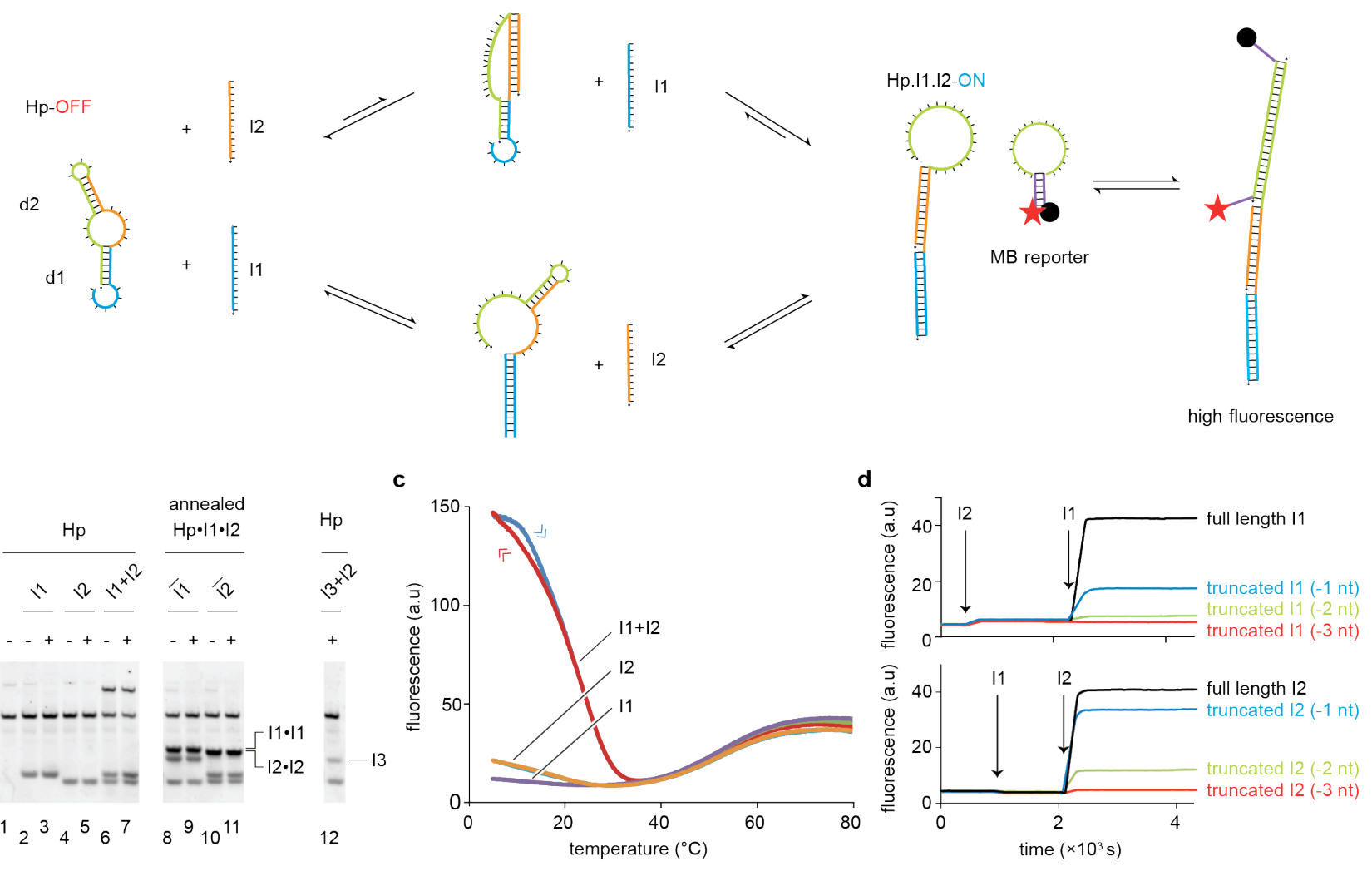

Figure 1. The reversible AND gate. a Designed network of interactions. b Gel analysis of the AND gate demonstrating cooperative binding of inputs I1 and I2 and the attainment of equilibrium under isothermal conditions. Samples were incubated for 30 minutes at $25^{\circ} \mathrm{C}$ or annealed (Supporting Information). $[\mathrm{Hp}]=100 \mathrm{nM}$, [Inputs] $=200 \mathrm{nM}$, [Input complements] $=400 \mathrm{nM}$. I3 is an input which shares the same displacement domain as I1, but has a different toehold. $\mathbf{c}$ Reporter fluorescence as a function of temperature, demonstrating thermodynamic reversibility and cooperativity of input binding. Samples were first cooled, then heated at a rate of $0.1^{\circ} \mathrm{C} / \mathrm{min}$. d Time-dependent reporter fluorescence demonstrating implementation of AND operation through cooperative binding of inputs. Defective inputs, truncated by 1,2 or 3 nt, are effectively discriminated against. Inputs were added where indicated. For $\mathbf{c}$ and $\mathbf{d},[\mathrm{Hp}]=500 \mathrm{nM}$, $[$ Reporter $]=500 \mathrm{nM}$, $[$ Inputs $]=1 \mu \mathrm{M}$.

were cleaved then the two inputs would bind independently. If the central loop were retained but the terminal loop cleaved then binding of $\mathrm{I} 2$ would be stabilized by opening the central loop (as in the case of I1), rather than destabilized by transforming it into the entropically more costly $\mathrm{d} 2$ loop: with appropriate reactant concentrations this would make it possible to engineer negative cooperativity between inputs.

Binding of the inputs is reversible. I1 and $\mathrm{I} 2$ are expected to bind with rate constants ${ }^{29,31}$ of $10^{4}-10^{6} \mathrm{M}^{-1} \mathrm{~s}^{-1}$, i.e. on a time scale of 1 $100 \mathrm{~s}$ under our experimental conditions. The displaced domain of Hp competes with I1 or I 2 through an intra-molecular displacement reaction: ${ }^{34}$ equilibration of $\mathrm{Hp}$ with the inputs is expected to occur on a similar time scale.

When $\mathrm{Hp}$ is in the ON state, it can bind reversibly to the reporter. The reporter has a stabilizing effect on Hp.I1.I2: Nupack predicts than in presence of all components (both inputs, the hairpin and the reporter) about $30 \%$ of $\mathrm{Hp}$ is in the form Hp.I1.I2.Reporter and $36 \%$ is in the form Hp.I1.I2.

The concentrations of free inputs I1 and I 2 are readily increased by adding these strands to the reaction; they can be decreased by adding complementary strands to sequester the inputs in stable duplexes. Figure 1b shows analysis by polyacrylamide gel electrophoresis (PAGE) of the cooperative and reversible interactions between the Hp AND gate and its inputs. The conformation of the reporter-binding domain of $\mathrm{Hp}$ changes significantly only when both inputs are present: it reverts to the OFF state when either of the inputs is sequestered. Incubation and annealing of reactants yield similar distributions of products, confirming that the state of Hp does not depend on its history.
Melting experiments presented in Figure 1c confirm that the gate operates as designed. Above $20^{\circ} \mathrm{C}$, no hysteresis is observed, indicating that thermodynamic equilibrium is maintained as the temperature is ramped up and down at a rate of $0.1{ }^{\circ} \mathrm{C} / \mathrm{min}$. Above $40^{\circ} \mathrm{C}$, the reporter beacon is unbound for all combinations of inputs: the increase of fluorescence with temperature corresponds to the opening of the reporter hairpin (Supporting Information). Below $40^{\circ} \mathrm{C}$, the fluorescence increases significantly as the temperature decreases if and only if $\mathrm{Hp}$ and both inputs are present. This increase results from binding of the reporter to $\mathrm{Hp}$ in the $\mathrm{ON}$ state.

Kinetic fluorescence experiments confirm that the reporter binds significantly to $\mathrm{Hp}$ if and only if both inputs are present (Figure 1d). A small increase in fluorescence ( $5 \%$ of the maximum signal) is observed when $\mathrm{I} 2$ only is added to $\mathrm{Hp}$, indicating that $\mathrm{I} 2$ can bind weakly to Hp in the absence of I1, as expected.

Reversibility confers tolerance to errors ${ }^{35}$. In DNA tile assem$\mathrm{bly}^{26}$, for example, a wrongly inserted tile can be locked in by the subsequent assembly of surrounding tiles, but the frequency of such kinetically trapped defects can be greatly reduced ${ }^{26}$ if assembly occurs close to the melting temperature of valid tile bindings. Kinetic traps are also observed in DNA secondary structures created during temperature jumps ${ }^{36}$. Figure 1 contains the results of tests of the robustness of the Hp gate to defective inputs. Fig. 1b lane 12 contains the products of annealing Hp with $\mathrm{I} 2$ and a modified input (I3) possessing the same displacement domain as I1 but a different toehold: no complex Hp.I3.I2 appears on the gel. Figure 1d includes the results of fluorescence experiments 
using inputs whose displacement domains are truncated by a few nucleotides (nt).
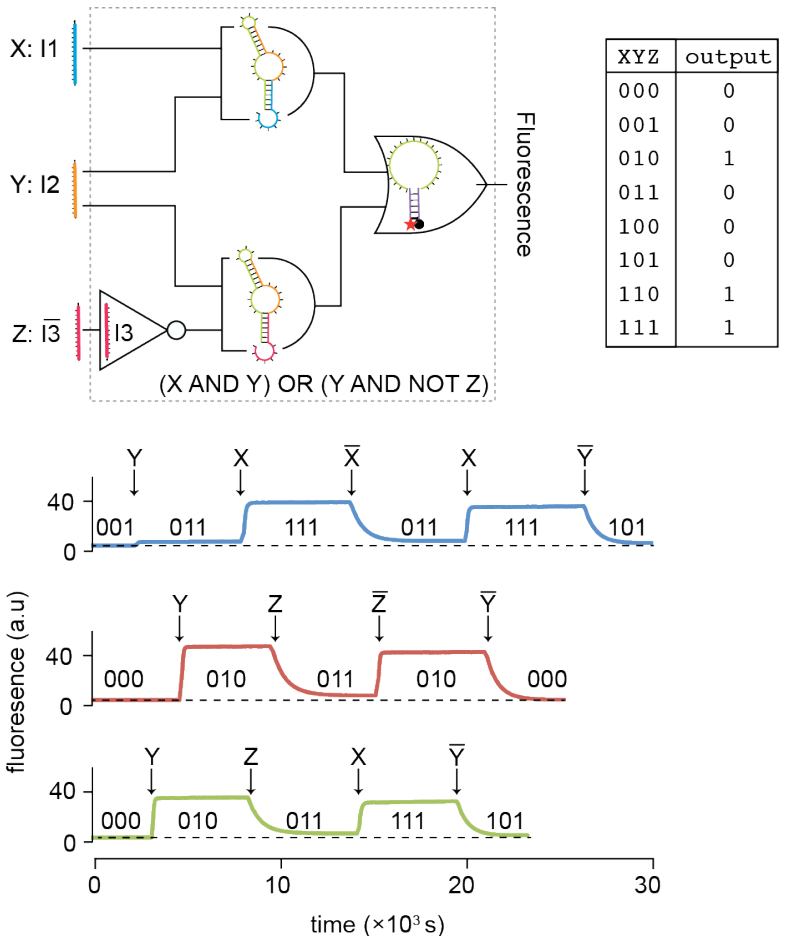

Figure 2. Response of a reversible logic circuit to changing inputs $X$ (first bit), Y (second bit) and $\mathrm{Z}$ (third bit). The NOT gate is implemented using a dual-rail convention, using hairpins which compute (I1 AND I2) and (I3 AND I2) and which interact with the same reporter. The circuit is preloaded with a stoichiometric amount of $\mathrm{I} 3$. Inputs are defined as: $\mathrm{X} \equiv \mathrm{I} 1 ; \mathrm{Y} \equiv \mathrm{I} 2$ and $\mathrm{Z} \equiv \overline{\mathrm{I} 3}$. Bits are changed by adding input strands or their complements, so that either strand is present at about $1 \mu \mathrm{M}$. Dotted horizontal lines indicate the baseline for each fluorescence trace.

The final fluorescence level decreases quickly with the magnitude of the defect: inputs truncated by $3 \mathrm{nt}$ do not noticeably switch $\mathrm{Hp}$ to the $\mathrm{ON}$ state. For gates operating under kinetic control, in contrast, an imperfect input can cause complete switching to the ON state, albeit at a reduced rate. ${ }^{37}$

Figure 2 demonstrates the operation of a Boolean circuit to compute (X AND Y) OR (X AND NOT Z). The OR operation is implemented by designing the two AND gates to expose the same domain $\mathrm{d} 2$ in their $\mathrm{ON}$ states. A dual-rail convention ${ }^{8,17}$ is used to implement a NOT gate: a stoichiometric amount of I3 is preloaded with the circuit (its concentration may be regarded as an 'internal variable') and $\overline{\mathrm{I} 3}$, the complement of $\mathrm{I} 3$, is defined as the external input $\mathrm{Z}$ to the circuit. Time-dependent fluorescence measurements are used to monitor the response of the circuit to changes in its inputs. In the upper trace, the third input $Z$ is kept constant at $1 \mu \mathrm{M}$. In the middle trace, the first bit $\mathrm{X}$ is kept constant at 0 . In the lower trace, all inputs vary. These experiments show that the circuit can change state several times in response to changes in its inputs, in contrast to circuits composed of irreversible gates which can be triggered once only. After the equilibration time (approx. 30 minutes) the state of the circuit depends only on its current inputs, not the input history. The fluorescent reporter also provides an upper bound on the switching time between the $\mathrm{ON}$ and OFF states (Figure 2): the half-time for switching from $\mathrm{OFF}$ to $\mathrm{ON}$ is less than $30 \mathrm{~s}$ and the half-time for switching from ON to OFF is less than $400 \mathrm{~s}$. These switching times include the binding or unbinding of the fluorescent reporter and are consistent with the results presented in Figure 1 and with the designed gate mechanism.

For useful biological applications, circuits may need additional layers of computation and to be able to handle arbitrary inputs. We have demonstrated two layers of computations, but the gates could, in principle, be cascaded further. The activated gate Hp$\mathrm{ON}$ is itself capable of strand displacement (Supporting Information), so could act as an input for a downstream gate. The base sequences of inputs to our gates are constrained by design, as with other logic circuits based on strand displacement. Translator gates $^{8,38}$ may be employed to transform an arbitrary input, such as a biological mRNA, into a signal compatible with the sequences of the gates. To maintain reversibility, the translator gates would themselves have to be reversible.

In summary, we have implemented reversible logic circuits whose outputs adjust to changes in the inputs. Cooperativity between inputs to the reversible AND gate is achieved by designed secondary structure linking the binding sites of the inputs. Reversible circuits could find applications beyond the reach of current irreversible systems in, e.g., interaction with dynamic systems such as oscillators ${ }^{39-41}$ that can be severely perturbed by monitoring reactions that consume reactants irreversibly ${ }^{41}$. Similar circuits could also provide enough computing power to monitor the expression of a few genes in real time. Gates based on this architecture could be directly expressed in-vivo as RNA transcripts ${ }^{42}$ or delivered $^{5,43}$ to cells.

\section{ASSOCIATED CONTENT}

Supporting Information Available: Materials and methods, further characterization of the gate, coupling of gates to irreversible strand displacement.

\section{AUTHOR INFORMATION}

\section{Corresponding Author}

a.turberfield@physics.ox.ac.uk

ACKNOWLEDGMENT A.J.G. was supported by: the Ecole Polytechnique, Paris; Merton College, Oxford; EPSRC grants EP/G037930/1 and EP/P503876/1.

\section{REFERENCES}

1. Beyer, S.; Simmel, F. C., Nucleic Acids Research 2006, 34 (5), 1581-1587

2. Rinaudo, K.; Bleris, L.; Maddamsetti, R.; Subramanian, S.; Weiss, R.; Benenson, Y., Nature Biotechnology 2007, 25 (7), 795801.

3. Modi, S.; Swetha, M. G.; Goswami, D.; Gupta, G. D.; Mayor, S.; Krishnan, Y., Nature Nanotechnology 2009, 4 (5), 325330 .

4. Graugnard, E.; Cox, A.; Lee, J.; Jorcyk, C.; Yurke, B.; Hughes, W. L., Ieee Transactions on Nanotechnology 2010, 9 (5), 603-609.

5. Choi, H. M. T.; Chang, J. Y.; Trinh, L. A.; Padilla, J. E.; Fraser, S. E.; Pierce, N. A., Nature Biotechnology 2010, 28 (11), 1208-U103.

6. Venkataraman, S.; Dirks, R. M.; Ueda, C. T.; Pierce, N. A., Proceedings of the National Academy of Sciences of the United States of America 2010, 107 (39), 16777-16782.

7. Benenson, Y.; Gil, B.; Ben-Dor, U.; Adar, R.; Shapiro, E., Nature 2004, 429 (6990), 423-429.

8. Seelig, G.; Soloveichik, D.; Zhang, D. Y.; Winfree, E., Science 2006, 314 (5805), 1585-1588.

9. Frezza, B. M.; Cockroft, S. L.; Ghadiri, M. R., Journal of the American Chemical Society 2007, 129 (48), 14875-14879. 
10. Zhang, D. Y.; Turberfield, A. J.; Yurke, B.; Winfree, E., Science 2007, 318 (5853), 1121-1125.

11. Ran, T.; Kaplan, S.; Shapiro, E., Nature Nanotechnology 2009, 4 (10), 642-648.

12. Takahashi, K.; Yaegashi, S.; Kameda, A.; Hagiya, M., Chain reaction systems based on loop dissociation of DNA. In DNA Computing, Carbone, A.; Pierce, N. A., Eds. 2006; Vol. 3892, pp 347-358.

13. Elbaz, J.; Lioubashevski, O.; Wang, F. A.; Remacle, F.; Levine, R. D.; Willner, I., Nature Nanotechnology 2010, 5 (6), $417-$ 422.

14. Pei, R. J.; Matamoros, E.; Liu, M. H.; Stefanovic, D.; Stojanovic, M. N., Nature Nanotechnology 2010, 5 (11), 773-777.

15. Lake, A.; Shang, S.; Kolpashchikov, D. M., Angewandte Chemie-International Edition 2010, 49 (26), 4459-4462.

16. Zhang, D. Y., Journal of the American Chemical Society 2011, 133 (4), 1077-1086.

17. Qian, L.; Winfree, E., Science 2011, 332, 1196-1201.

18. Qian, L. L.; Winfree, E.; Bruck, J., Nature 2011, 475 (7356), 368-372.

19. Seelig, G.; Yurke, B.; Winfree, E., Journal of the American Chemical Society 2006, 128 (37), 12211-12220.

20. Qian, L. L.; Winfree, E., Science 2011, 332 (6034), 11961201.

21. Soloveichik, D.; Seelig, G.; Winfree, E., Proceedings of the National Academy of Sciences of the United States of America 2010, 107 (12), 5393-5398.

22. Goel, A.; Ibrahimi, M., Natural Computing 2011, 10 (1), 467-485.

23. Bennett, C. H., Ibm Journal of Research and Development 1973,17 (6), 525-532.

24. Qian, L. L.; Soloveichik, D.; Winfree, E. In Efficient Turing-universal computation with DNA polymers., DNA 16, Hong Kong, Sakakibara, Y.; Mi, Y., Eds. Hong Kong, 2010.

25. Chiniforooshan, E.; Doty, D.; Kari, L.; Seki, S. In Scalable, Time-Responsive, Digital, Energy-Efficient Molecular Circuits using DNA Strand Displacement, Proceedings of the 16th International Meeting on DNA Computing and Molecular Programming, SpringerVerlag: 2010.

26. Barish, R. D.; Schulman, R.; Rothemund, P. W. K.; Winfree, E., Proceedings of the National Academy of Sciences of the United States of America 2009, 106 (15), 6054-6059.
27. Cardelli, L.; Laneve, C., Reversible structures. To appear in the proceedings of the 9th International Conference on Computational Methods in Systems Biology(CMSB 2011), 2011.

28. Tyagi, S.; Kramer, F. R., Nature Biotechnology 1996, 14 (3), 303-308

29. Zhang, D. Y.; Winfree, E., Journal of the American Chemical Society 2009, 131 (47), 17303-17314.

30. Yurke, B.; Turberfield, A. J.; Mills, A. P.; Simmel, F. C.; Neumann, J. L., Nature 2000, 406 (6796), 605-608.

31. Green, S. J.; Lubrich, D.; Turberfield, A. J., Biophysical Journal 2006, 91 (8), 2966-2975.

32. Zadeh, J. N.; Steenberg, C. D.; Bois, J. S.; Wolfe, B. R.; Pierce, M. B.; Khan, A. R.; Dirks, R. M.; Pierce, N. A., Journal of Computational Chemistry 2011, 32 (1), 170-173.

33. Gultyaev, A. P.; Van Batenburg, F. H. D.; Pleij, C. W. A., Rna-a Publication of the Rna Society 1999, 5 (5), 609-617.

34. Genot, A. J.; Zhang, D. Y.; Bath, J.; Turberfield, A. J., Journal of the American Chemical Society 2011, 133 (7), 2177-2182.

35. Whitesides, G. M.; Boncheva, M., Proceedings of the National Academy of Sciences of the United States of America 2002, 99 (8), 4769-4774

36. Viasnoff, V.; Meller, A.; Isambert, H., Nano Letters 2006, $6(1), 101-104$.

37. Zhang, D. Y.; Winfree, E., Nucleic Acids Research 2010, 38 (12), 4182-4197.

38. Picuri, J. M.; Frezza, B. M.; Ghadiri, M. R., Journal of the American Chemical Society 2009, 131 (26), 9368-9377.

39. Montagne, K.; Plasson, R.; Sakai, Y.; Fujii, T.; Rondelez, Y., Molecular Systems Biology 2011, 7.

40. Kim, J.; Winfree, E., Molecular Systems Biology 2011, 7.

41. Franco, E.; Friedrichs, E.; Kim, J.; Jungmann, R.; Murray, R.; Winfree, E.; Simmel, F. C., Proceedings of the National Academy of Sciences of the United States of America 2011, 108 (40), E784E793.

42. Delebecque, C. J.; Lindner, A. B.; Silver, P. A.; Aldaye, F. A., Science 2011, 333 (6041), 470-474

43. Walsh, A. S.; Yin, H.; Erben, C. M.; Wood, M. J. A.; Turberfield, A. J., Acs Nano 2011, 5 (7), 5427-5432. 
Graphical Table of Content

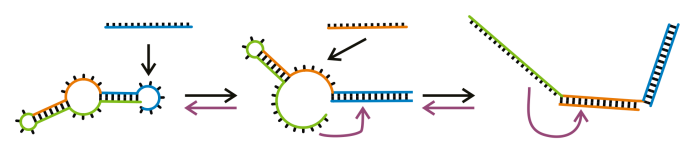

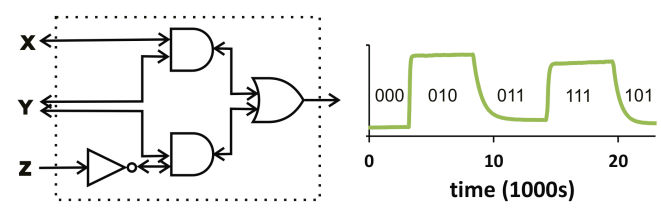

